\title{
Graphene: Free electron scattering within an inverted honeycomb lattice
}

\author{
Z. M. Abd El-Fattah, ${ }^{1,2, *}$ M. A. Kher-Elden, ${ }^{2}$ I. Piquero-Zulaica, ${ }^{3,4}$ F. J. García de Abajo, ${ }^{1,5, \dagger}$ and J. E. Ortega ${ }^{3,4,6, \ddagger}$ \\ ${ }^{1}$ ICFO-Institut de Ciencies Fotoniques, The Barcelona Institute of Science and Technology, 08860 Castelldefels (Barcelona), Spain \\ ${ }^{2}$ Physics Department, Faculty of Science, Al-Azhar University, Nasr City, E-11884 Cairo, Egypt \\ ${ }^{3}$ Centro de Física de Materiales (CSIC-UPV-EHU) and Materials Physics Center (MPC), 20018 San Sebastián, Spain \\ ${ }^{4}$ Donostia International Physics Center, Paseo Manuel Lardizábal 4, E-20018 Donostia-San Sebastián, Spain \\ ${ }^{5}$ ICREA-Institució Catalana de Recerca i Estudis Avançats, Passeig Lluís Companys 23, 08010 Barcelona, Spain \\ ${ }^{6}$ Departamento de Física Aplicada I, Universidad del País Vasco, 20018 San Sebastián, Spain
}

(Received 13 December 2018; revised manuscript received 28 February 2019; published 29 March 2019)

\begin{abstract}
Theoretical progress in graphene physics has largely relied on the application of a simple nearest-neighbor tight-binding model capable of predicting many of the electronic properties of this material. However, important features that include electron-hole asymmetry and the detailed electronic bands of basic graphene nanostructures (e.g., nanoribbons with different edge terminations) are beyond the capability of such a simple model. Here we show that a similarly simple plane-wave solution for the one-electron states of an atom-based two-dimensional potential landscape, defined by a single fitting parameter (the scattering potential), performs better than the standard tight-binding model, and levels to density-functional theory in correctly reproducing the detailed $\pi$-band structure of a variety of graphene nanostructures. In particular, our approach identifies the three hierarchies of nonmetallic armchair nanoribbons, as well as the doubly-degenerate flat bands of free-standing zigzag nanoribbons with their energy splitting produced by symmetry breaking. The present simple plane-wave approach holds great potential for gaining insight into the electronic states and electro-optical properties of graphene nanostructures and other two-dimensional materials with intact or gapped Dirac-like dispersions.
\end{abstract}

DOI: 10.1103/PhysRevB.99.115443

\section{INTRODUCTION}

The two-dimensional (2D) honeycomb carbon-atom lattice known as graphene [1] is a promising material for applications in optical and electronic devices [2-4]. In particular, its unique conical electronic dispersion $[5,6]$ and 2D character enable an exceptionally large optical tunability $[7,8]$ and configure a suitable playground for quantum electrodynamics phenomena, such as the relativistic Klein tunneling [9], as well as a customizable zoo of exotic band structures when decorated with defects [10], arranged in twisted bilayers [11], or laterally patterned into ribbons $[12,13]$. Energy-gap engineering in graphene-an essential prerequisite for nanoelectronics applications - demands controlled and selective sublattice perturbations at the atomic scale, such as those one can produce through chemical doping [14,15], electrical gating [16], lateral strain [17,18], and substrate-induced sublattice asymmetry [19-22].

Graphene nanoribbons (GNRs) have been extensively studied as simple, appealing nanostructures that lead to electronic band features of interest, such as gap openings, due to quantum confinement and peculiar edge states that can readily be tuned through their width, shape, and edge terminations $[12,13]$. Rapid progress in on-surface chemistry, which allows controlled synthesis of novel graphene-based nanostructures, such as GNRs with complex architectures [23-28], combined

\footnotetext{
*Corresponding author: z.m.abdelfattah@azhar.edu.eg

${ }^{\dagger}$ Corresponding author: javier.garciadeabajo@nanophotonics.es

‡Corresponding author: enrique.ortega@ehu.es
}

with the precise mapping of their electronic structures using angle-resolved photoemission spectroscopy (ARPES) and scanning tunneling spectroscopy (STS) [29-32], render GNRs as promising candidates for the realization of exotic graphenebased nanodevices [33-35].

Theoretical understanding and prediction of extended graphene and GNRs properties has been instrumental in the development of the field. Density-functional theory (DFT) accurately describes their electronic structures, but simpler methods [36-39] are preferred because they allow us to gain further physical insight. In particular, following the pioneering work of Wallace [39], the tight-binding (TB) model has played a central role in the theoretical description of the electronic structure of extended graphene, yielding remarkable agreement with DFT calculations. However, noticeable discrepancies between TB and DFT show up when describing GNRs with either armchair (AGNR) or zigzag (ZGNR) edge terminations. For example, the widely used nearest-neighbors TB predicts two families of AGNRs, namely semiconductor and metallic, depending on the number of carbon-dimer lines along the ribbon width $\left(N_{a}\right)$ [13], while three semiconductor categories are obtained from DFT calculations [40] in agreement with STS experiments [41-44]. These discrepancies, which stem from the fact that the nearest-neighbors TB model renormalizes long-range interactions and is only strictly applicable to extended graphene, can be resolved for nanoribbons at the expense of including at least third-neighbors hoppings $[45,46]$, in addition to electron-electron interaction in ZGNRs, for example at the level of a mean-field Hubbard- $U$ term $[47,48]$. 
Both nearest-neighbors TB and nearly-free electron (NFE) models are well-known textbook approaches for bandstructure calculations in solids [49,50]. Within the NFE framework, plane wave expansions (PWEs) of the electron states have traditionally played an important role, for example in the description of electron scattering in metallic and molecular superlattices [51-53]. In particular, 2D hexagonal superlattices, which are known to exhibit graphenelike band structures with $\bar{M}$-point gap and symmetry-protected degeneracy at the $\bar{K}$ points [54,55], are well described by the PWE approach. Unfortunately, such simple PWEs have not been used for the description of extended graphene or GNRs, although a close correspondence between the TB and NFE models was demonstrated for the so-called molecular graphene, in which the Shockley surface state confined by a hexagonal CO superlattice was shown to exhibit a Dirac-like dispersion [56].

Here, we demonstrate that the electronic characteristics of $\pi$ bands in atomic graphene can be finely reproduced via a simple NFE model with a single fitting parameter, namely the scattering potential. In this context, the graphene non-Bravais honeycomb lattice is alternatively modeled as a $2 \mathrm{D}$ hexagonal lattice made of the sixfold symmetric, hexagonally-warped inner part of the carbon rings, where a sufficiently large repulsive potential $V_{3}$ is assigned [Fig. 1(a)]. The potential barrier $V_{3}$ in reality delimits the attractive Coulomb potential of each carbon atom $\left(V_{1}\right.$ and $\left.V_{2}\right)$. Perfect agreement with DFT calculations is obtained for the band structure, local density of states (LDOS), and constant-energy surfaces (CESs) using an electron-plane-wave-expansion (EPWE) implementation (see Sec. II). Interestingly, with the same single fitting parameter $V_{3}$, the model captures the three categories of AGNRs in decent agreement with DFT. Likewise, the 1D-bulk band structure and the nearly-degenerate edge state for ZGNRs are obtained in agreement with TB and DFT without any inclusion of electron-electron interactions. Additionally, we find that when the symmetry of the two carbon sublattices is broken for ZGNRs $\left(V_{1} \neq V_{2}\right)$, which is a common situation for graphene grown onto different substrates, the edge state of ZGNRs is split in energy, also without the incorporation of electron-electron interactions. We believe that this simplified picture can be efficiently applied to explore different varieties of atomic graphenelike extended and finite structures.

\section{THEORETICAL FORMALISM}

\section{A. Effective 2D potential description}

We simulate the electronic structure of graphene in terms of the one-electron states of a 2D potential landscape, in which each carbon atom is represented by a circle filled with uniform potential, embedded in a flat interstitial region [see Fig. 1(a)]. We then write the Schrödinger equation as

$$
-\frac{\hbar^{2}}{2 m_{\mathrm{eff}}} \nabla^{2} \phi+V \phi=E \phi
$$

where the energy $E$ is expressed relative to a reference level (e.g., the Dirac point), $m_{\text {eff }}$ is the effective mass, $V(\mathbf{R})$ is the 2D potential as a function of spatial coordinates $\mathbf{R}=(x, y)$ along the graphene plane, and $\phi(\mathbf{R})$ is the electron wave function.
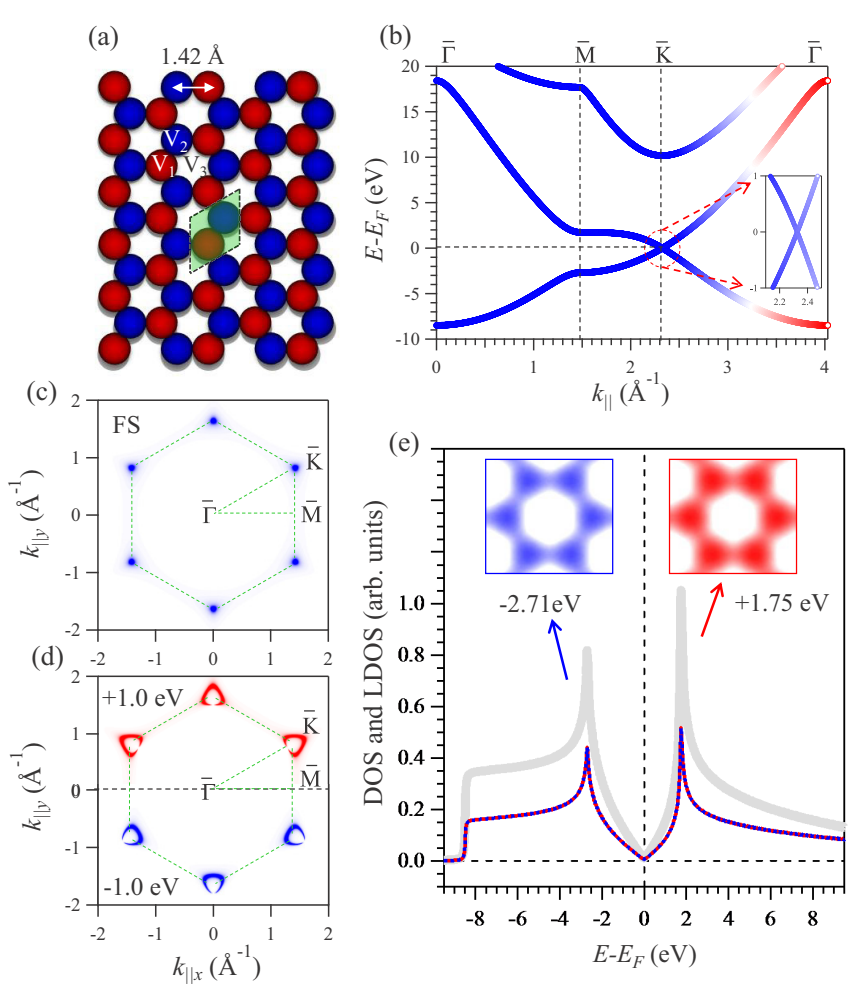

FIG. 1. Free-standing graphene. (a) Geometry and scattering potential used in our EPWE calculations. The red and blue circles represent the two carbon sublattices with inner potentials $V_{1}$ and $V_{2}$, while white regions define the hexagonally-warped bases with an inner potential $V_{3}$. (b) Calculated band structure using EPWE along the $\overline{\Gamma M K \Gamma}$ excursion within the first Brillouin zone (BZ), for $V_{3}=23 \mathrm{eV}$ and $V_{1}=V_{2}=0$. The inset is a closeup view at the $\bar{K}$ point. (c),(d) Simulated CESs taken at (c) $0 \mathrm{eV}$ and at (d) $+1 \mathrm{eV}$ (top half) and $-1 \mathrm{eV}$ (bottom half). (e) LDOS calculated at the center of the carbon atoms in each sublattice (red and blue) and total DOS per unit cell (gray). The insets show the 2D LDOS at the boundaries of the $\bar{M}$-point gap.

\section{B. Plave-wave expansion for periodic systems}

We take $V$ to be periodic and express it in terms of Fourier components as

$$
V(\mathbf{R})=\sum_{\mathbf{g}} V_{\mathbf{g}} \mathrm{e}^{\mathrm{i} \mathbf{g} \cdot \mathbf{R}},
$$

where the sum extends over 2D reciprocal lattice vectors $\mathbf{g}$ with coefficients $V_{\mathbf{g}}$ calculated as an integral over the unit cell (UC), normalized to the unit-cell area $A$ :

$$
V_{\mathbf{g}}=\frac{1}{A} \int_{\mathrm{UC}} d^{2} \mathbf{R} V(\mathbf{R}) \mathrm{e}^{-\mathrm{i} \cdot \mathbf{R}} .
$$

Using Bloch's theorem, we anticipate electron wave functions labeled by a band index $j$ and the $2 \mathrm{D}$ wave vector $\mathbf{k}_{\|}$within the first Brillouin zone (1BZ):

$$
\phi_{\mathbf{k}_{\| j} j}(\mathbf{R})=\frac{1}{\sqrt{N A}} \sum_{\mathbf{g}} \phi_{\mathbf{k}_{\|} j, \mathbf{g}} \mathrm{e}^{\mathrm{i}\left(\mathbf{k}_{\|}+\mathbf{g}\right) \cdot \mathbf{R}},
$$

where we use the (infinite) number of cells $N$ for normalization purposes. Inserting Eqs. (2) and (4) into Eq. (1), we find 
the linear system of equations

$E_{\mathbf{k}_{\| j} j} \phi_{\mathbf{k}_{\|} j, \mathbf{g}}=\sum_{\mathbf{g}^{\prime}}\left[\left(\hbar^{2} / 2 m_{\mathrm{eff}}\right)\left|\mathbf{k}_{\|}+\mathbf{g}\right|^{2} \delta_{\mathbf{g}, \mathbf{g}^{\prime}}+V_{\mathbf{g}-\mathbf{g}^{\prime}}\right] \phi_{\mathbf{k}_{\|} j, \mathbf{g}^{\prime}}$

Because $V_{\mathbf{g}-\mathbf{g}^{\prime}}$ is a Hermitian matrix with indices $\mathbf{g}$ and $\mathbf{g}^{\prime}$, for each value of $\mathbf{k}_{\|}$we obtain different bands $j$ of real eigenenergies $E_{\mathbf{k}_{\| j}}$ and eigenstates of coefficients $\phi_{\mathbf{k}_{\| j}, \mathbf{g}}$. We solve Eq. (5) by retaining a finite number of g's within a sufficiently large distance $g_{\max }$ to the origin in reciprocal space (i.e., $\left.|\mathbf{g}| \leqslant g_{\max }\right)$. The eigenstates form an orthonormal and complete system, that is,

$$
\begin{aligned}
\int d^{2} \mathbf{R} \phi_{\mathbf{k}_{\|} j}(\mathbf{R}) \phi_{\mathbf{k}_{\|}^{\prime} j^{\prime}}^{*}(\mathbf{R}) & =\delta_{\mathbf{k}_{\|} \mathbf{k}_{\|}^{\prime}} \delta_{j j^{\prime}}, \\
\sum_{\mathbf{k}_{\|} j} \phi_{\mathbf{k}_{\|} j}(\mathbf{R}) \phi_{\mathbf{k}_{\| j}}^{*}\left(\mathbf{R}^{\prime}\right) & =\delta\left(\mathbf{R}-\mathbf{R}^{\prime}\right),
\end{aligned}
$$

provided we impose the normalization condition $\sum_{\mathbf{g}}\left|\phi_{\mathbf{k}_{\|} j, \mathbf{g}}\right|^{2}=1$. The integral in Eq. (6a) is extended over the entire 2D plane.

For extended graphene, good convergence (indistinguishable results within the scale of graphs) is achieved with $g_{\max }=$ $7 / \sqrt{A}$, while for nanoribbons $g_{\max }$ needs to be increased to $20 / \sqrt{A}$. In both cases, the number of plane waves involved in the calculation scales as $g_{\max }^{2}$.

\section{LDOS calculation for periodic systems}

The local density of states (LDOS) is directly calculated from its definition

$$
\operatorname{LDOS}(E, \mathbf{R})=2 \sum_{\mathbf{k}_{\|} j}\left|\phi_{\mathbf{k}_{\|} j}(\mathbf{R})\right|^{2} \delta\left(E-E_{\mathbf{k}_{\|} j}\right),
$$

where the factor of 2 accounts for spin degeneracy. In practice, we use the prescription $\sum_{\mathbf{k}_{\|}} \rightarrow\left(N A / 4 \pi^{2}\right) \int d^{2} \mathbf{k}_{\|}$and evaluate this integral in a dense wave-vector grid by interpolating the eigenstates and eigenenergies within each grid element.

\section{Calculation of photoemission angular distributions for periodic systems}

In order to make the calculation feasible and simple, we dismiss the contribution of the normal component of the electron wave function to the photoemission matrix elements, as it should just introduce a smooth and broad angular dependence, which we represent through a multiplicative coefficient $C$ in the resulting photoemission intensity. We focus instead on the contribution of the in-plane wave function of the initial electron state and further approximate the parallel component of the photoelectron wave function as a normalized plane wave $\mathrm{e}^{\mathrm{i} \mathbf{k}_{\|}^{\text {out }} \cdot \mathbf{R}} / \sqrt{N A}$ of outgoing parallel wave vector $\mathbf{k}_{\|}^{\text {out }}$. The doubly-differential angle-resolved photoemission intensity corresponding to a binding energy $E$ and photoelectron wave vector $\mathbf{k}_{\|}^{\text {out }}$ is then given from Fermi's golden rule as

$$
\begin{aligned}
\frac{d I(E)}{d^{2} \mathbf{k}_{\|}^{\text {out }}} & =C \sum_{\mathbf{k}_{\|} j}\left|\int d^{2} \mathbf{R} \phi_{\mathbf{k}_{\| j} j}(\mathbf{R}) \frac{\mathrm{e}^{-\mathrm{i} \mathbf{k}_{\|}^{\text {out }} \cdot \mathbf{R}}}{\sqrt{N A}}\right|^{2} \delta\left(E-E_{\mathbf{k}_{\| j} j}\right) \\
& =\left.C \sum_{j, \mathbf{g}}\left|\phi_{\mathbf{k}_{\|} j, \mathbf{g}}\right|^{2} \delta\left(E-E_{\mathbf{k}_{\|} j}\right)\right|_{\mathbf{k}_{\|}=\mathbf{k}_{\|}^{\text {out }}-\mathbf{g}},
\end{aligned}
$$

where the sum over $\mathbf{g}$ in the last expression is restricted to reciprocal vectors such that $\mathbf{k}_{\|}=\mathbf{k}_{\|}^{\text {out }}-\mathbf{g}$ lies within the $1 \mathrm{BZ}$.

\section{RESULTS AND DISCUSSION}

Figure 1 summarizes the electronic characteristics of freestanding graphene, as determined within the EPWE approach. The potential landscape used in the calculations is depicted in Fig. 1(a). The red and blue circles define the position of the carbon atoms, each of radius $0.71 \AA$ (i.e., nearest neighbors are tangentially touching at one point without overlap), and the white regions stand for the carbon-free interstitial region. The unit cell, enclosing two carbon atoms surrounded by the interstitial region, is marked by the black dashed lines (shaded area), with unit-cell vectors of length $d=\sqrt{3} a_{0}$, where $a_{0}=$ $1.42 \AA$ is the $\mathrm{C}-\mathrm{C}$ bond distance in the material. The band structure presented in Fig. 1(b) is obtained by setting the interstitial potential to $V_{3}=23 \mathrm{eV}$ and the two equivalent carbonatom potentials to $V_{1}=V_{2}=0$, so the potential difference is $\Delta V=23 \mathrm{eV}$. Furthermore, we take the effective mass $m_{\mathrm{eff}}$ equal to the electron mass. These parameters successfully reproduce the band structure of free-standing graphene obtained from DFT calculations and experiments [57,58]. The Fermi energy $E_{\mathrm{F}}$ is set at the nongapped (see zoom-in) Dirac point, and the $\bar{\Gamma}$-point energy is accordingly found at $\sim-8.5 \mathrm{eV}$. The lower and upper edges of the $\bar{M}$-point gap are $-2.71 \mathrm{eV}$ and $+1.75 \mathrm{eV}$, respectively, while the slope of the linear bands at the $\bar{K}$ points is $\approx 6.5 \mathrm{eV} \AA$ (i.e., approximately $\hbar$ times the Fermi velocity $\left.v_{\mathrm{F}} \approx 10^{6} \mathrm{~m} / \mathrm{s}\right)$, in excellent agreement with literature values [59]. We stress that small deviations from the employed values of $\Delta V$ and $m_{\text {eff }}$ yield noticeable changes in the relative energy position of the bands, the size of the $\bar{M}$-point gap, and the degree of electron-hole asymmetry [see Fig. S1 in the Supplemental Material (SM)] [60].

In Figs. 1(c) and 1(d) we present the simulated photoemission intensity of the constant energy surfaces (CESs). The Fermi surface (FS) consists of single spots centered at the six $\bar{K}$ points of the BZ (green hexagon), resulting from intact Dirac cones [Fig. 1(c)]. At lower and higher binding energies (e.g., $+1 \mathrm{eV}$ and $-1 \mathrm{eV}$ ), these spots diverge into the characteristic graphene triangular pockets, as shown in the upper (red) and lower (blue) panels of Fig. 1(d), respectively. The trigonal shape of these CESs further confirms that the electron-hole asymmetry present in (b), which in TB calculations is accounted for by introducing additional hopping parameters for second and third nearest neighbors $[6,61,62]$, is naturally captured by EPWE. Indeed, the different hopping parameters employed in TB are consistent with the different effective potentials (e.g., height $\times$ width) felt by electrons moving across neighboring atoms. Furthermore, the variation of the photoemission intensity within the trigonal pockets agrees nicely with recent ARPES experiments [20,63-65]. The electronic characteristics of graphene, as deduced from the total density of states (DOS) and LDOS, are also presented in Fig. 1(e). The V-like profiles at $E_{\mathrm{F}}$ are revealed in both the DOS (gray) and the LDOS evaluated at the two carbon atoms (blue and red), all of them exhibiting a clear electron-hole asymmetry. The onset of the LDOS at $\sim-8.5 \mathrm{eV}$ defines the $\bar{\Gamma}$-point energy, whereas the peaks at $-2.71 \mathrm{eV}$ and $+1.75 \mathrm{eV}$ signal the borders of the $\bar{M}$-point gap. The LDOS at the 


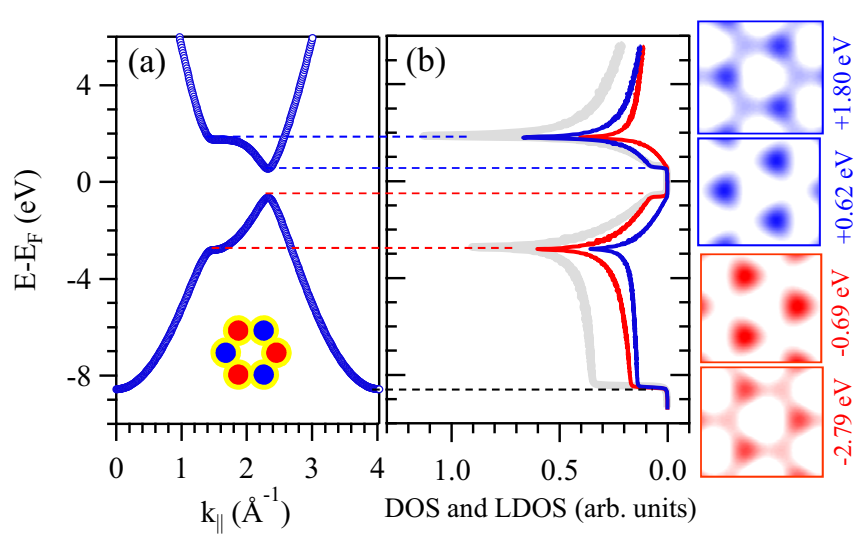

FIG. 2. Perturbed graphene. (a) Calculated band structure using EPWE along the $\overline{\Gamma M K \Gamma}$ excursion for $V_{3}=23 \mathrm{eV}, V_{1}=+1 \mathrm{eV}$, and $V_{2}=-1 \mathrm{eV}$. (b) The corresponding DOS (gray) and LDOS taken at the red and blue circles in (a). The right panel in (b) presents twodimensional LDOS maps taken at the lower (red) and upper (blue) edges of the $\bar{M}$-point and $\bar{K}$-point gaps.

two carbon atoms are coincident, as they clearly must in pristine graphene. The 2D LDOS maps depicted at insets and taken at the boundaries of the $\bar{M}$-point gap are equally identical, confirming the absence of $\bar{K}$-point gaps based on symmetry considerations [54,55]. Given the calculations and analysis presented in Fig. 1, the electronic features of a freestanding graphene sheet obtained from experiments and DFT calculations are well reproduced by EPWE.

Perturbations induced by a graphene support (i.e., a substrate and/or the deposition of adsorbates/dopants) have been shown to change the electronic properties of graphene in different ways. Figure 2 presents possible electronic modifications in one of such perturbation schemes, as calculated using the present EPWE approach. In Fig. 2(a), we explore the effect of broken symmetry between the two carbon atoms on the electronic band structure. This is obtained by assigning different potentials to each of the carbon sublattices $\left[V_{1}=-1 \mathrm{eV}\right.$ (red) and $V_{2}=+1 \mathrm{eV}$ (blue)]. The main modification consists of the opening of an energy gap at the $\bar{K}$ point $\left[E_{g}(\bar{K})=\right.$ $1.3 \mathrm{eV}$ ], which is a natural consequence of broken symmetry in the potential landscape within the unit cell. Such brokensymmetry-induced gaps have been reported experimentally for different graphene structures, such as graphene grown on $\operatorname{Ir}(111)$ [66], Ru(0001) [67], hydrogenated-graphene [68], and other systems [19-22,65]. The large gap induced by broken symmetry shown here is meant only to illustrate the effect, but the actual values of $V_{1}$ and $V_{2}$ can in principle be tuned in each case to yield the experimental gap. We also note that the $\bar{\Gamma}$-point energy is unaltered in this particular example to first order, as the average of the potentials $V_{1}$ and $V_{2}$ is zero. The DOS and LDOS spectra presented in (b) precisely follow these band structure modifications. In addition to the peaks at the borders of the $\bar{M}$-point gap, a deviation from the V-shape profiles occurs for all spectra, where instead the DOS and LDOS vanish at the energy range spanning the $\bar{K}$-point gap boundaries. Particularly relevant is the nonequivalence of the LDOS at the two carbon atoms within the unit cell, where the weight of the LDOS changes from one carbon (red) to the second sublattice (blue) by crossing the energy gap, which is further shown in the 2D LDOS maps presented in Fig. 2(b) (right panels). Additional electronic modifications, such as hybridization and doping, which still preserve the $\bar{K}$-point degeneracy, are briefly presented in Fig. S2 of the SM [60].

The calculations and analysis here presented clearly reveal that the electronic structure of graphene could be reproduced through a relatively simple $2 \mathrm{D}$ potential landscape with specific values of the potential in different regions of space. In what follows, we explore the applicability of this approach to the study of both AGNRs and ZGNRs. We employ the same geometry and potential landscape while varying the ribbon width and termination. We make use of the periodic supercell approach and assume the ribbons to be infinitely extended along their axis (i.e., the $x$ direction), while they are decoupled in the $y$ direction by separating them by $\geqslant 20 \AA$ gaps. The periodicity along the ribbon is given by $d$ and $a=3 a_{0}$ for ZGNR and AGNR, respectively. Figures 3(a)-3(c) depict the band structure along the ribbon axis for the three different classes of AGNRs with (a) $N_{a}=3 p$, (b) $N_{a}=3 p+1$, and (c) $N_{a}=$ $3 p+2$, where $p$ is a positive integer [here, $p=1$ (left) and 7 (right)]. In contrast to standard TB calculations, the three types of ribbons exhibit energy gaps $E_{g}$ satisfying the relation $E_{g}(3 p+1)>E_{g}(3 p)>E_{g}(3 p+2)$, with sizes following an inverse proportionality with the GNR width [see colored curves in Fig. 3(d)] in agreement with DFT calculations. It should be noted that the carbon-carbon distance in the bulk region and at the ribbon boundaries is fixed here to the same value (i.e., structural relaxation is not considered, although DFT indicates a $\sim 3.5 \%$ contraction in the bond length at the edges) [40], yet the model yields appreciable energy gaps for the $3 p+2$ family. We also show that the $\bar{\Gamma}$-point energy, irrespectively of the ribbon family, asymptotically approaches the $\bar{\Gamma}$ point of extended graphene by increasing the width, strictly following the width-dependent $\bar{\Gamma}$ point energy (gray curve) obtained by stepping along the Brillouin zone slices of graphene [see inset in Fig. 3(d)]. Particularly relevant is the shallow dispersion of the bands at $-2.6 \mathrm{eV}$ for $N_{a}=3$ (a) and $N_{a}=5$ (c), which in nearest-neighbor TB exhibit no dispersion $[13,69]$. This constitutes a further indication that the NFE approach naturally incorporates all possible crosstalk and hoppings between neighboring carbon atoms with the scattering potential as a single fitting parameter.

In what follows, we check the ability of our model to simulate the photoemission intensity and DOS for nanoribbons. This should serve as guidance for experimentalists to perform proper assignments of specific GNR bands, which might become problematic for wider ribbons with neighboring dispersion bands, and in general, due to strong variations in intensity caused by effects related to the photoemission matrix elements. Figure 3(e) presents the simulated photoemission intensity for the 4-AGNR along the ribbon axis. A subtle variation of photoemission intensity for different bands is clear. For example, at $k_{y}=0$ (left) the frontier of the valence band (VB1) has predominantly-symmetric spectral weight around $k_{x}=0$, while VB2 gains spectral weight over a wider $k_{x}$ range with an asymmetric photoemission intensity around the top of the band $\left(\sim 1.5 \AA^{-1}\right)$. This distribution of photoemission intensity changes drastically at $k_{y}=\pi / a$ (center) and $k_{y}=$ $2 \pi / a$ (right). Indeed, these are all 1D bands, and therefore, 

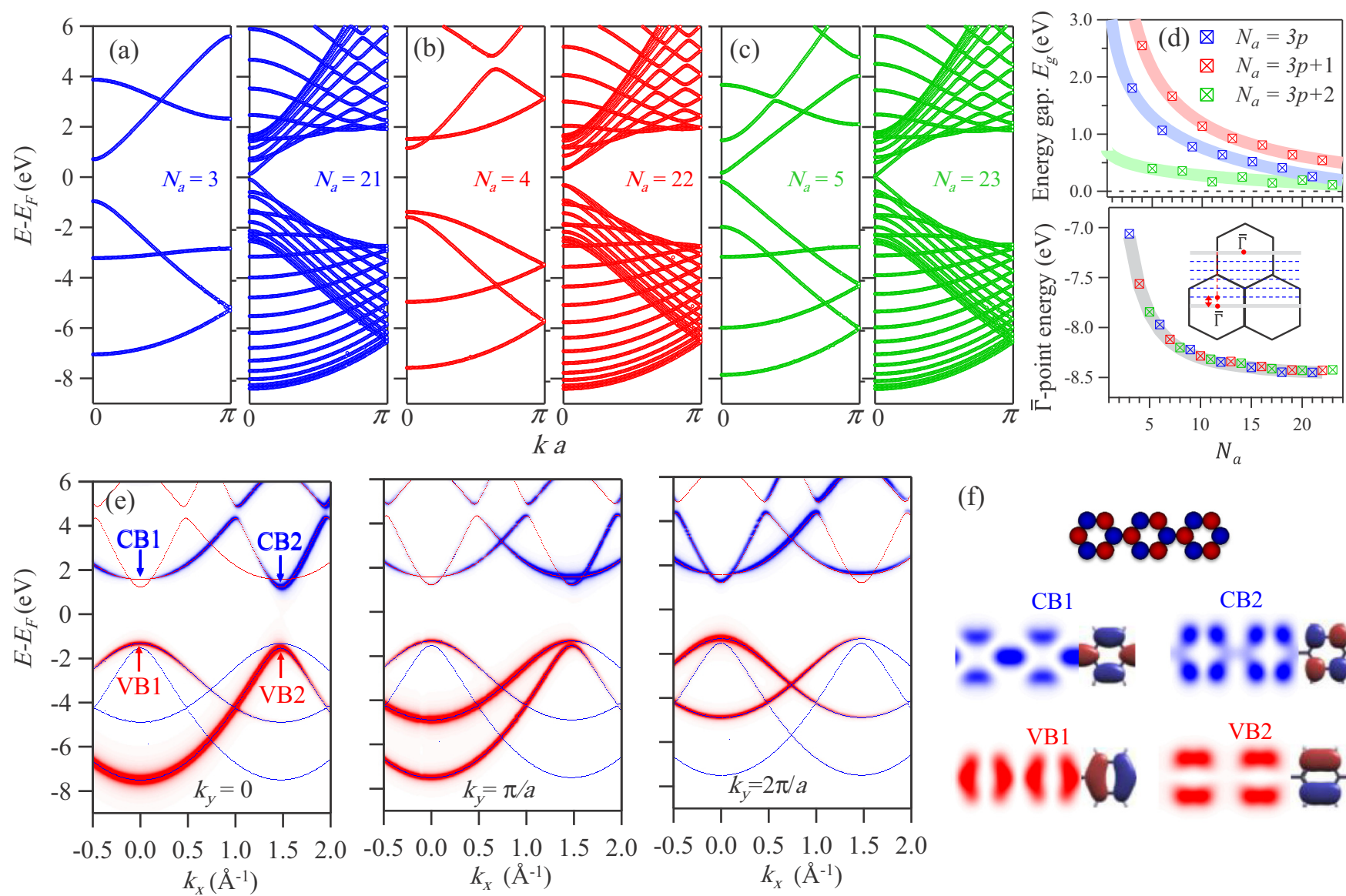

(f)
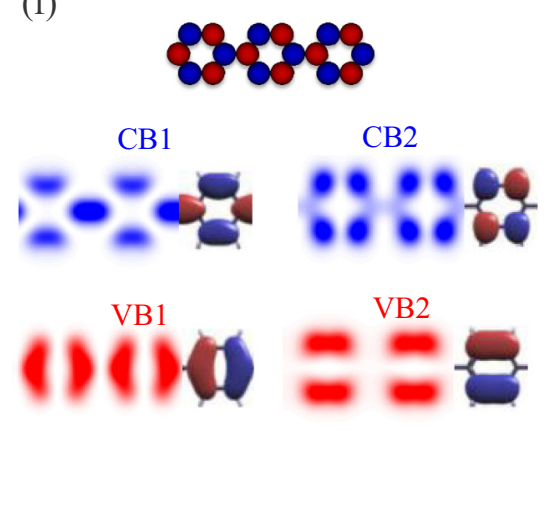

FIG. 3. Armchair graphene nanoribbons (AGNRs). (a)-(c) Band structure of $N_{a}$-AGNR with $N_{a}=3$ and 21 (a), 4 and 22 (b), and 5 and 23 (c), as obtained using EPWE. (d) Variation of the gap size and $\bar{\Gamma}$-point energy for the three categories of AGNRs. The inset shows the first and second BZs of graphene. (e) Simulated photoemission intensity for the 4-AGNR along the ribbon axis ( $k_{x}$ direction) taken at $k_{y}=0$ (left), $k_{y}=\pi / a$ (center), and $k_{y}=2 \pi / a$ (right). (f) LDOS for the 3-AGNR taken at valence- and conduction-band points VB1, VB2, CB1, and CB2 [see labels in (e)]. The corresponding molecular orbitals deduced from DFT calculations are shown to the right (adapted from Ref. [71]), and the geometry used in EPWE simulations is shown at the top.

not dispersing in the perpendicular direction $k_{y}$, yet a strong modulation of the photoemission intensity is present for the VB and conduction band (CB) alike [Fig. S3(a), SI] [60]. Therefore, constant-energy surfaces (i.e., $k_{x}$ vs $k_{y}$ maps) such as the one shown in Fig. S3(d) of the SM [60] are essential for a proper assignment of bands. Actually, the simulation of such photoemission intensity maps has recently solved a long-standing contradiction between STS and ARPES data for the 7-AGNR, where the VB2 point was mistakenly assigned in ARPES experiments to VB1 [30,70]. A further confirmation of the full functionality of our model is provided by comparing the calculated 2D LDOS maps to the molecular orbitals obtained from DFT calculations, and in particular, for the 3-AGNR, as shown in Fig. 3(f). The matching between LDOS (left) and DFT orbitals (right) is remarkable [71]. The overall agreement with DFT extends even beyond the description of simple AGNRs: Complex graphene-based structures such as zigzag [71] and heterogeneous ribbons, as well as nanoporous graphene [28], are equally well described using our NFE approach (see Fig. S4, SM) [60].

Likewise, the electronic structure of ZGNRs could be obtained using our EPWE approach. Figures 4(a) and 4(b) present band structure calculations for selected ZGNRs with
$N_{a}=12$ (a) and 6 (b). Their characteristic bulk bands and the energy-degenerate edge states are obtained in agreement with TB and DFT calculations when on-site Hubbard potentials and exchange interactions, respectively, are not considered. In (b) the simulated photoemission intensity for the $6-Z G N R$ is also shown at $k_{y}=0$, with the edge state exhibiting a high-enough spectral weight as to be probed by average techniques such as ARPES, provided that the ZGNRs aligned on substrates are experimentally available over large areas.

As previously discussed, for an extended graphene sheet with symmetry-broken carbon sublattices, a $\bar{K}$-point gap opens up (see Fig. 2). Here we demonstrate the effect that this broken symmetry has on the electronic structure of ZGNRs, specifically on the edge state. By assigning different values to the potentials at the two carbons sublattices $\left(V_{1}=-1 \mathrm{eV}\right.$ and $V_{2}=+1 \mathrm{eV}$ ), taking the 12-ZGNR as an example, we show that the edge state is split in energy, while the 1Dbulk projected bands are practically unaltered [Fig. 4(c)]. The energy gap between the split edge states is the same as the size of the $\bar{K}$-point gap of the corresponding extended graphene presented in Fig. 2(a). Both bands of the energy-split edge state have reasonable photoemission spectral weights, 

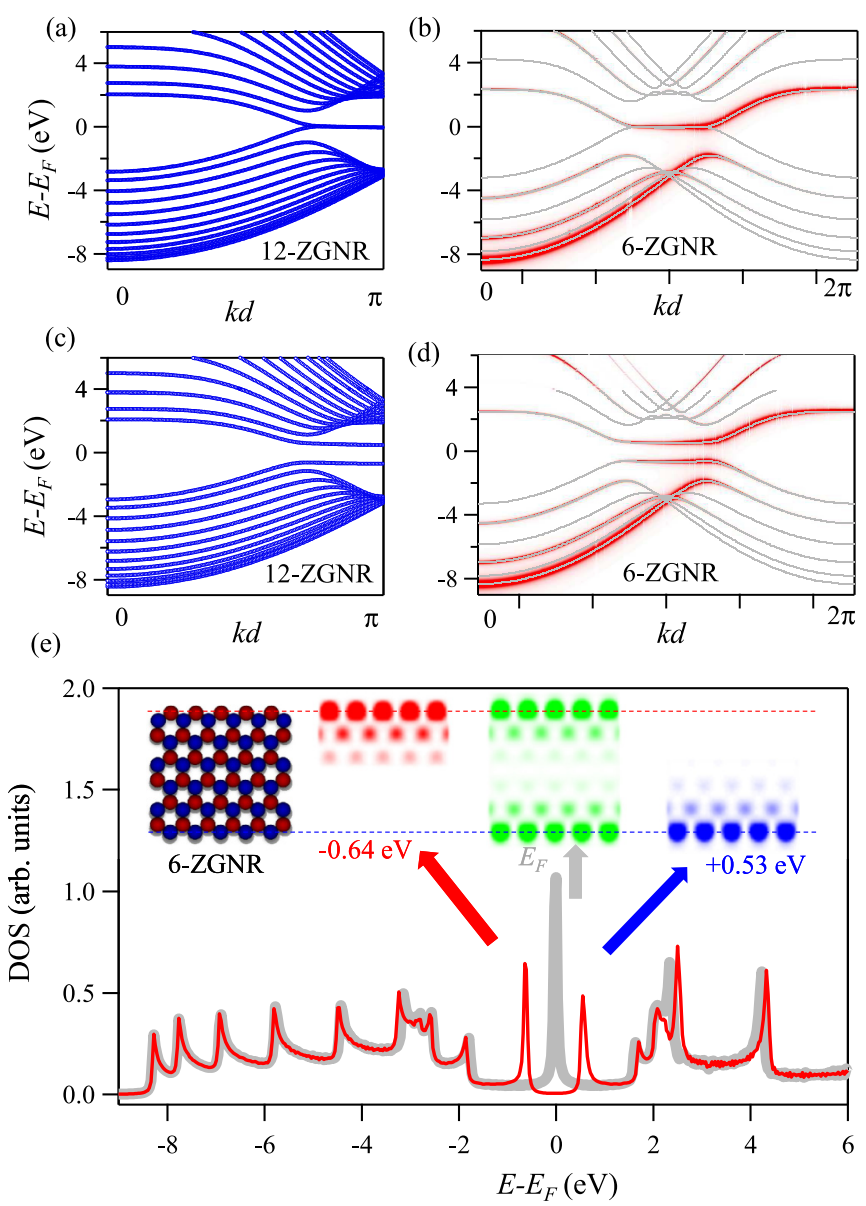

FIG. 4. Zigzag graphene nanoribbons (ZGNRs). (a-b) Band structure of 12-ZGNR (a) and 6-ZGNR (b) obtained using EPWE. $(c, d)$ Same as $(a, b)$, but assuming that the symmetry of the carbon sublattice is now broken. The simulated photoemission intensity for the 6-ZGNR with an in-plane photoelectron momentum along the ribbon and $k_{y}=0$ is shown in (b,d). (e) DOS for the 6-ZGNR before (gray) and after (red) breaking the symmetry of the carbon sublattices. The insets show the atomic structure and two-dimensional LDOS maps at the edge-state energies.

as demonstrated for the 6-ZGNR at $k_{y}=0$ in Fig. 4(d). Finally, we present in Fig. 4(e) the DOS curves for the free-standing (gray) and symmetry-broken (red) sublattices in the 6-ZGNR, where energy splitting of the DOS peak at $E_{\mathrm{F}}$ is observed. Although the DOS profile for asymmetric ribbons (red) resembles the one reported experimentally (and from DFT) for the 6-ZGNR [72], the broken-symmetryinduced gaps could be distinguished from electron-electroninteraction gaps $[40,73,74]$ by plotting the $2 \mathrm{D}$ spatial distribution of the LDOS taken at the energy of the edge state, as shown in insets above the spectra in Fig. 4(e). These insets present the EPWE geometry of the 6-ZGNR and the 2D LDOS maps taken at the energy of the lower (red) and upper (blue) edge states, where the LDOS is clearly localized at one edge, while for fully symmetric ribbons both edges are equally occupied at $E_{\mathrm{F}}$ (green). It should be noted that the oneedge localization of the LDOS has not been experimentally reported, neither for degenerate nor for energy-split edges states, although its realization could have potential impact as a switch in $1 \mathrm{D}$ conduction channels through gating. We also anticipate that the combination of broken symmetry and electron correlation should produce a clear imbalance in the LDOS at both edges of the ribbons, in addition to the intrinsic asymmetry produced by the different dispersion of the upper and lower edges of the gap and the electron-hole asymmetry.

The fact that the extended and finite graphene characteristics are well captured within the framework of the NFE model could have far reaching implications, since some of the electronic structure variations and size dependence are not unique to graphene. Similar atomic systems, such as silicene or boron nitride, could be understood following the same approach. Furthermore, nanometer-sized ribbons made from hexagonal superlattices, such as molecular graphene [56] or metallic superlattices possessing graphenelike band structures [54], should exhibit these types of size-dependent variations. What makes these variations experimentally accessible and potentially relevant for technology in both graphene and other types of 2D crystals is the combination of a large $\bar{M}$-point gap (several electronvolts, which for superlattices reduces to just a few meV) and the steep dispersion near the $\bar{K}$ points. Finally, this simple NFE description of graphene and its nanostructures should have a large impact on the efficient simulation of graphene-based devices and phenomena, such as negative refraction and super lenses in $p$ - $n$ junctions, using, for example, the complementary electronic boundaryelement method (EBEM) solver, which was previously used to describe similar effects in $2 \mathrm{D}$ metallic superlattices $[75,76]$.

\section{CONCLUSIONS}

We have shown that the electronic structure of the $\pi$ band in free-standing and perturbed graphene can be reasonably well described using a simple nearly-free-electron model (the EPWE approach) applied to an inverted honeycomb lattice that is defined by a sufficiently large confining potential. With a single fitting parameter (i.e., the scattering barrier) the electronic properties of common graphene nanostructures (armchair and zigzag graphene nanoribbons) are successfully described using this simple model. Our approach simplifies the exploration of newly emerging artificial systems with fundamental and technological interest, such as nanostructured 2D materials, topological GNR junctions with peculiar end states [26,27], and artificial flat band lattices [77].

\section{ACKNOWLEDGMENTS}

This work has been supported in part by the Spanish MINECO (Grants No. MAT2014-59096-P, No. MAT2016-78293-C6, No. MAT-2017-88374-P, No. MAT201788492-R, and No. SEV2015-0522), the Basque Government (Grant No. IT-1255-19), the European Research Council (Advanced Grant No. 789104-eNANO), the European Commission (Graphene Flagship 696656), the Catalan CERCA Program, Fundació Privada Cellex, and AGAUR (Grant No. 2017 SGR 1651). 
[1] A. K. Geim and K. S. Novoselov, Nat. Mater. 6, 183 (2007).

[2] M. Liu, X. Yin, E. Ulin-Avila, B. Geng, T. Zentgraf, L. Ju, F. Wang, and X. Zhang, Nature (London) 474, 64 (2011).

[3] L. Vicarelli, M. S. Vitiello, D. Coquillat, A. Lombardo, A. C. Ferrari, W. Knap, M. Polini, V. Pellegrini, and A. Tredicucci, Nat. Mater. 11, 4176 (2012).

[4] F. Schwierz, Nat. Nanotechnol. 5, 487 (2010).

[5] G. W. Semenoff, Phys. Rev. Lett. 53, 2449 (1984).

[6] A. H. Castro Neto, F. Guinea, N. M. R. Peres, K. S. Novoselov, and A. K. Geim, Rev. Mod. Phys. 81, 109 (2009).

[7] Z. Fei, A. S. Rodin, G. O. Andreev, W. Bao, A. S. McLeod, M. Wagner, L. M. Zhang, Z. Zhao, M. Thiemens, G. Dominguez et al., Nature (London) 487, 82 (2012).

[8] J. Chen, M. Badioli, P. Alonso-González, S. Thongrattanasiri, F. Huth, J. Osmond, M. Spasenović, A. Centeno, A. Pesquera, P. Godignon et al., Nature (London) 487, 77 (2012).

[9] M. I. Katselson, K. S. Novoselov, and A. K. Geim, Nat. Phys. 2, 620 (2006).

[10] C. Forsythe, X. Zhou, K. Watanabe, T. Taniguchi, A. Pasupathy, P. Moon, M. Koshino, P. Kim, and C. R. Dean, Nat. Nanotechnol. 13, 566 (2018).

[11] Y. Cao, V. Fatemi, S. Fang, K. Watanabe, T. Taniguchi, E. Kaxiras, and P. Jarillo-Herrero, Nature (London) 556, 43 (2018).

[12] M. Fujita, K. Wakabayashi, K. Nakada, and K. Kusakabe, J. Phys. Soc. Jpn. 65, 1920 (1996).

[13] K. Nakada, M. Fujita, G. Dresselhaus, and M. S. Dresselhaus, Phys. Rev. B 54, 17954 (1996).

[14] F. Joucken, Y. Tison, P. L. Fèvre, A. Tejeda, A. Taleb-Ibrahimi, V. R. E. Conrad, C. Chacon, A. Bellec, Y. Girard, S. Rousset et al., Sci. Rep. 5, 14564 (2015).

[15] I. Gierz, C. Riedl, U. Starke, C. R. Ast, and K. Kern, Nano Lett. 8, 4603 (2008).

[16] N. M. R. Peres, Rev. Mod. Phys. 82, 2673 (2010).

[17] Z. H. Ni, T. Yu, Y. H. Lu, Y. Y. Wang, Y. P. Feng, and Z. X. Shen, ACS Nano 2, 2301 (2008).

[18] M. Conrad, F. Wang, M. Nevius, K. Jinkins, A. Celis, M. Narayanan Nair, A. Taleb-Ibrahimi, A. Tejeda, Y. Garreau, A. Vlad et al., Nano Lett. 17, 341 (2017).

[19] S. Y. Zhou, G.-H. Gweon, A. V. Fedorov, P. N. First, W. A. de Heer, D.-H. Lee, F. Guinea, A. H. Castro Neto, and A. Lanzara, Nat. Mater. 6, 770 (2007).

[20] H. Vita, S. Böttcher, K. Horn, E. N. Voloshina, R. E. Ovcharenko, T. Kampen, A. Thissen, and Y. S. Dedkov, Sci. Rep. 4, 5704 (2014).

[21] M. Papagno, S. Rusponi, P. M. Sheverdyaeva, S. Vlaic, M. Etzkorn, D. Pacilé, P. Moras, C. Carbone, and H. Brune, ACS Nano 6, 199 (2012).

[22] A. Varykhalov, D. Marchenko, J. Sánchez-Barriga, M. R. Scholz, B. Verberck, B. Trauzettel, T. O. Wehling, C. Carbone, and O. Rader, Phys. Rev. X 2, 041017 (2012).

[23] Y.-C. Chen, T. Cao, C. Chen, Z. Pedramrazi, D. Haberer, D. G. de Oteyza, F. R. Fischer, S. G. Louie, and M. F. Crommie, Nat. Nanotechnol. 10, 156 (2015).

[24] M. Di Giovannantonio, O. Deniz, J. I. Urgel, R. Widmer, T. Dienel, S. Stolz, C. Sánchez-Sánchez, M. Muntwiler, T. Dumslaff, R. Berger et al., ACS Nano 12, 74 (2018).

[25] C. Bronner, R. A. Durr, D. J. Rizzo, Y.-L. Lee, T. Marangoni, A. M. Kalayjian, H. Rodriguez, W. Zhao, S. G. Louie, F. R. Fischer et al., ACS Nano 12, 2193 (2018).
[26] O. Gröning, X. Y. Sh. Wang, C. A. Pignedoli, G. B. Barin, C. Daniels, A. Cupo, V. Meunier, X. Feng, A. Narita, K. Müllen et al., Nature (London) 560, 209 (2018).

[27] D. J. Rizzo, G. Veber, T. Cao, C. Bronner, T. Chen, F. Zhao, H. Rodriguez, S. G. Louie, M. F. Crommie, and F. R. Fischer, Nature (London) 560, 204 (2018).

[28] C. Moreno, M. Vilas-Varela, B. Kretz, A. Garcia-Lekue, M. V. Costache, M. Paradinas, M. Panighel, G. Ceballos, S. O. Valenzuela, D. Peña et al., Science 360, 199 (2018).

[29] P. Ruffieux, J. Cai, N. C. Plumb, L. Patthey, D. Prezzi, A. Ferretti, E. Molinari, X. Feng, K. Müllen, C. A. Pignedoli et al., ACS Nano 6, 6930 (2012).

[30] B. V. Senkovskiy, D. Y. Usachov, A. V. Fedorov, D. Haberer, N. Ehlen, F. R. Fischer, and A. Grüneis, 2D Mater. 5, 035007 (2018).

[31] E. Carbonell-Sanromá, A. Garcia-Lekue, M. Corso, G. Vasseur, P. Brandimarte, J. Lobo-Checa, D. G. de Oteyza, J. Li, S. Kawai, S. Saito et al., J. Phys. Chem. C 122, 16092 (2018).

[32] G. Z. Magda, X. Jin, I. Hagymási, P. Vancsó, Z. Osváth, P. Nemes-Incze, C. Hwang, L. P. Biró, and L. Tapasztó, Nature (London) 514, 608 (2014).

[33] T. Ihn, J. Güttinger, F. Molitor, S. Schnez, E. Schurtenberger, A. Jacobsen, S. Hellmüller, T. Frey, S. Dröscher, C. Stampfer et al., Mater. Today 13, 44 (2010).

[34] J. P. Llinas, A. Fairbrother, G. Borin Barin, W. Shi, K. Lee, S. Wu, B. Y. Choi, R. Braganza, J. Lear, N. Kau et al., Nat. Commun. 8, 633 (2017).

[35] A. Celis, M. N. Nair, A. Taleb-Ibrahimi, E. H. Conrad, C. Berger, W. A. de Heer, and A. Tejeda, J. Phys. D: Appl. Phys. 49, 143001 (2016).

[36] M. V. Fischetti, J. Kim, S. Narayanan, Z. Y. Ong, C. Sachs, D. K. Ferry, and Sh. J. Aboud, J. Phys.: Condens. Matter 25, 473202 (2013).

[37] P. Marconcini and M. Macucci, La Rivista del Nuovo Cimento 34, 489 (2011).

[38] G. S. Kissinger and S. Satpathy, Phys. Rev. B 94, 205126 (2016).

[39] P. R. Wallace, Phys. Rev. 71, 622 (1947).

[40] Y.-W. Son, M. L. Cohen, and S. G. Louie, Nature (London) 444, 348 (2006).

[41] A. Kimouche, M. M. Ervasti, R. Drost, S. Halonen, A. Harju, P. M. Joensuu, J. Sainio, and P. Liljeroth, Nat. Commun. 6, 10177 (2015).

[42] N. Merino-Díez, A. Garcia-Lekue, E. Carbonell-Sanromá, J. Li, M. Corso, L. Colazzo, F. Sedona, D. Sánchez-Portal, J. I. Pascual, and D. G. de Oteyza, ACS Nano 11, 11661 (2017).

[43] H. Söde, L. Talirz, O. Gröning, C. A. Pignedoli, R. Berger, X. Feng, K. Müllen, R. Fasel, and P. Ruffieux, Phys. Rev. B 91, 045429 (2015).

[44] Y. Li, M. Chen, M. Weinert, and L. Li, Nat. Commun. 5, 4311 (2014).

[45] C. T. White, J. Li, D. Gunlycke, and J. W. Mintmire, Nano Lett. 7, 825 (2007).

[46] D. Gunlycke and C. T. White, Phys. Rev. B 77, 115116 (2008).

[47] L. Pisani, J. A. Chan, B. Montanari, and N. M. Harrison, Phys. Rev. B 75, 064418 (2007).

[48] D. Gunlycke, D. A Areshkin, J. Li, J. W. Mintmire, and C. T. White, Nano Lett. 7, 3608 (2007). 
[49] C. Kittel, Quantum theory of solids (Wiley, New York, 1987).

[50] N. W. Ashcroft and N. D. Mermin, Solid State Physics (Harcourt College Publishers, Philadelphia, 1976).

[51] A. Mugarza, A. Mascaraque, V. Pérez-Dieste, V. Repain, S. Rousset, F. J. García de Abajo, and J. E. Ortega, Phys. Rev. Lett. 87, 107601 (2001).

[52] J. E. Ortega and F. J. García de Abajo, Nat. Nanotechnol. 2, 601 (2007).

[53] I. Piquero-Zulaica, J. Lobo-Checa, A. Sadeghi, Z. M. Abd ElFattah, C. Mitsui, T. Okamoto, R. Pawlak, T. Meier, A. Arnau, J. E. Ortega et al., Nat. Commun. 8, 787 (2017).

[54] D. Malterre, B. Kierren, Y. Fagot-Revurat, C. Didiot, F. J. García de Abajo, F. Schiller, J. Cordón, and J. E. Ortega, New J. Phys. 13, 013026 (2011).

[55] Z. M. Abd El-Fattah, M. Matena, M. Corso, F. J. García de Abajo, F. Schiller, and J. E. Ortega, Phys. Rev. Lett. 107, 066803 (2011).

[56] K. K. Gomes, W. Mar, W. Ko, F. Guinea, and H. C. Manoharan, Nature (London) 483, 306 (2012).

[57] P. Puschnig and D. Lüftner, J. Electr. Spectrosc. Relat. Phenom. 200, 193 (2015).

[58] A. A. Rybkina, A. G. Rybkin, A. V. Fedorov, D. Y. Usachov, M. E. Yachmenev, D. E. Marchenko, O. Y. Vilkov, A. V. Nelyubov, V. K. Adamchuk, and A. M. Shikin, Surf. Sci. 609, 7 (2013).

[59] Y. Dedkov and E. Voloshina, J. Phys.: Condens. Matter 27, 303002 (2015).

[60] See Supplemental Material at http://link.aps.org/supplemental/ 10.1103/PhysRevB.99.115443 for additional information on the electron-hole asymmetry, graphene-substrate hybridization, doped-graphene, detailed photoemission intensity, and the band structure of heterogeneous AGNR and nanoporous graphene.

[61] A. Kretinin, G. L. Yu, R. Jalil, Y. Cao, F. Withers, A. Mishchenko, M. I. Katsnelson, K. S. Novoselov, A. K. Geim, and F. Guinea, Phys. Rev. B 88, 165427 (2013).

[62] S. Reich, J. Maultzsch, C. Thomsen, and P. Ordejón, Phys. Rev. B 66, 035412 (2002).

[63] A. V. Fedorov, N. I. Verbitskiy, D. Haberer, C. Struzzi, L. Petaccia, D. Usachov, O. Y. Vilkov, D. V. Vyalikh, J. Fink, M. Knupfer et al., Nat. Commun. 5, 3257 (2014).

[64] S. Ulstrup, P. Lacovig, F. Orlando, D. Lizzit, L. Bignardi, M. Dalmiglio, M. Bianchi, F. Mazzola, A. Baraldi, R. Larciprete et al., Surf. Sci. 678, 57 (2018).
[65] A. Varykhalov, J. Sánchez-Barriga, A. M. Shikin, C. Biswas, E. Vescovo, A. Rybkin, D. Marchenko, and O. Rader, Phys. Rev. Lett. 101, 157601 (2008).

[66] M. Kralj, I. Pletikosić, M. Petrović, P. Pervan, M. Milun, A. T. N'Diaye, C. Busse, T. Michely, J. Fujii, and I. Vobornik, Phys. Rev. B 84, 075427 (2011).

[67] T. Brugger, S. Günther, B. Wang, J. H. Dil, M.-L. Bocquet, J. Osterwalder, J. Wintterlin, and T. Greber, Phys. Rev. B 79, 045407 (2009).

[68] R. Balog, B. Jørgensen, L. Nilsson, M. Andersen, E. Rienks, M. Bianchi, M. Fanetti, E. Lægsgaard, A. Baraldi, S. Lizzit et al., Nat. Mater. 9, 315 (2010).

[69] G. Vasseur, Y. Fagot-Revurat, M. Sicot, B. Kierren, L. Moreau, D. Malterre, L. Cardenas, G. Galeotti, J. Lipton-Duffin, F. Rosei et al., Nat. Commun. 7, 10235 (2016).

[70] Vicinal surfaces are frequently used for ARPES measurement because they help to align graphene nanoribbons and define the $k_{x}$ direction. However, the nanoribbon plane could be tilted a few degrees with respect to the vicinal surface plane, leading to an uncertain normal emission geometry and hence to a sizable $k_{y}$ projection. For example, using a $\alpha=5^{\circ}$ vicinal surface and He I excitation energy $(h v=21.2 \mathrm{eV}), k_{y}$ could vary by as much as $0.51 \times \sqrt{h v-\phi} \times \sin \alpha \sim 0.2 \AA^{-1}$, where $\phi$ stands for the work function, leading to a confusing assignment of the detected nanoribbon bands.

[71] I. Piquero-Zulaica, A. Garcia-Lekue, L. Colazzo, C. K. Krug, M. Sabri, Z. M. Abd El-Fattah, J. M. Gottfried, D. G. de Oteyza, J. E. Ortega, and J. Lobo-Checa, ACS Nano 12, 10537 (2018).

[72] P. Ruffieux, S. Wang, B. Yang, C. Sánchez-Sánchez, J. Liu, T. Dienel, L. Talirz, P. Shinde, C. A. Pignedoli, D. Passerone et al., Nature (London) 531, 489 (2016).

[73] Y. W. Son, M. L. Cohen, and S. G. Louie, Phys. Rev. Lett. 97, 216803 (2006).

[74] L. Yang, M. L. Cohen, and S. G. Louie, Phys. Rev. Lett. 101, 186401 (2008).

[75] F. J. García de Abajo, J. Cordón, M. Corso, F. Schiller, and J. E. Ortega, Nanoscale 2, 717 (2010).

[76] Z. M. Abd El-Fattah, M. A. Kher-Elden, O. Yassin, M. M. ElOkr, J. E. Ortega, and F. J. García de Abajo, J. Appl. Phys. 122, 195306 (2017).

[77] D. Leykam, A. Andreanov, and S. Flach, Adv. Phys.: X 3, 1473052 (2018). 